\title{
Group theoretical approach to the intertwined Hamiltonians
}

\author{
José F. Cariñena and Arturo Ramos \\ Departamento de Física Teórica. Facultad de Ciencias. \\ Universidad de Zaragoza, 50009, Zaragoza, Spain. \\ E-mail: jfc@posta.unizar.es and arrg@wigner.unizar.es \\ and
}

David J. Fernández C.

Departamento de Física, CINVESTAV-IPN, A.P. 14-740, 07000 México D.F., México.

E-mail: david@fis.cinvestav.mx

\begin{abstract}
We show that the finite difference Bäcklund formula for the Schrödinger Hamiltonians is a particular element of the transformation group on the set of Riccati equations considered by two of us in a previous paper. Then, we give a group theoretical explanation to the problem of Hamiltonians related by a first order differential operator. A generalization of the finite difference algorithm relating eigenfunctions of three different Hamiltonians is found, and some illustrative examples of the theory are analyzed, finding new potentials for which one eigenfunction and its corresponding eigenvalue is exactly known.
\end{abstract}

Key Words: Finite difference Bäcklund algorithm, intertwining technique, $A-$ related Hamiltonians, transformation group of Riccati equations, solvable potentials.

\section{INTRODUCTION}

The factorization of Schrödinger Hamiltonians in terms of differential operators of first order plays an important rôle in the search of quantum systems for which the energy spectrum is completely known $[1,2,3]$. It is closely related with the existence of intertwining operators $[4,5], \mathrm{Su}-$ persymmetric Quantum Mechanics [6] and Darboux transformations [7], among other questions. Indeed, most of the exactly solvable potentials can be obtained by making use of an appropriate intertwining operator transformation. However, nowadays the point is not just to factorize some 
well-known Schrödinger Hamiltonians [1] but to generate new solvable ones. The key to this technique is to intertwine two different Hamiltonians by a differential operator $A$, usually of first order $[3,4,5,8,9,10]$ or higher $[11,12,13,14,15,16,17,18]$, provided one of them is solvable. Concerning iterations of the first order intertwining technique, one of the authors (DJF), Hussin and Mielnik [4] have recently used a finite difference algorithm which provides algebraically the solution of the key Riccati equation at a given iteration step in terms of two solutions of the corresponding Riccati equation at the previous step associated to two different 'factorization energies'. This procedure has been successfully applied in order to obtain new exactly solvable Hamiltonians departing from the harmonic oscillator and Coulomb potentials $[4,5,19]$.

On the other hand, a number of authors have studied the transformation groups related with Riccati equations [20, 21, 22, 23, 24, 25, 26, 27]. In the last of these references, it has been used a transformation group on the set of Riccati equations in order to analyze their integrability. We think that this group theoretic approach could shed light on the abovementioned problem of intertwined or $A$-related Hamiltonians. A natural question is whether there is a relation of this group action with the finite difference algorithm.

We will try to answer these questions in this article, which is organized as follows. In Section 2 we recall briefly the problem of two Hamiltonians intertwined by a first order differential operator, a technique which is also known as $A$-related Hamiltonians. In Section 3 we relate the Schrödinger equations arising in the previous problem with certain Riccati equations by making use of the classical Lie theory of reduction by infinitesimal symmetries of differential equations, as applied to homogeneous linear secondorder equations. In Section 4 we recall the action of a group on the set of Riccati equations described in [27]. Using this technique we will prove the finite difference theorem [4] in a way alternative to [28]. In Section 5 we will determine the elements of the transformation group preserving the subset of Riccati equations arising from the set of Schrödinger equations after applying the reduction process outlined in Section 3. We will find a new transformation relating three different Schrödinger equations, which represents a generalization of the finite difference Bäcklund algorithm. In Section 6 we find that the problem of $A$-related Hamiltonians can be explained exactly in terms of the transformation group on the set of Riccati equations and the reduction procedure of Section 3. Section 7 illustrates the use of the new Theorems of Section 5 in the search of potentials for which one eigenstate and the corresponding eigenvalue will be exactly known. In particular, Examples 7.1, 7.3 and 7.4 will provide potentials essentially different from the original ones. Finally, we give in Section 8 some conclusions and an outlook for future work. 


\section{HAMILTONIANS RELATED BY FIRST-ORDER DIFFERENTIAL OPERATORS.}

The simplest way of generating an exactly solvable Hamiltonian $\widetilde{H}$ from a known one $H$ is just to consider an invertible bounded operator $B$, with bounded inverse, and defining $\widetilde{H}=B H B^{-1}$. This transformed Hamiltonian $\widetilde{H}$ has the same spectrum as the starting one $H$. As a generalization (see e.g. [3]), we will say that two Hamiltonians $H$ and $\widetilde{H}$ are intertwined or $A$-related when $A H=\widetilde{H} A$, where $A$ may have no inverse. In this case, if $\psi$ is an eigenvector of $H$ corresponding to the eigenvalue $E$ and $A \psi \neq 0$, at least formally $A \psi$ is also an eigenvector of $\widetilde{H}$ corresponding to the same eigenvalue $E$.

If $A$ is a first order differential operator,

$$
A=\frac{d}{d x}+W(x), \quad \text { and } \quad A^{\dagger}=-\frac{d}{d x}+W(x),
$$

then the relation $A H=\widetilde{H} A$, with

$$
H=-\frac{d^{2}}{d x^{2}}+V(x), \quad \widetilde{H}=-\frac{d^{2}}{d x^{2}}+\widetilde{V}(x),
$$

lead to

$$
V=-2 W^{\prime}+\widetilde{V}, \quad W(V-\widetilde{V})=-W^{\prime \prime}-V^{\prime} .
$$

Taking into account the first equation, the second becomes $2 W W^{\prime}=W^{\prime \prime}+$ $V^{\prime}$, which can easily be integrated giving

$$
V=W^{2}-W^{\prime}+\epsilon
$$

and then,

$$
\widetilde{V}=W^{2}+W^{\prime}+\epsilon,
$$

where $\epsilon$ is an integration constant. The important point here is that $H$ and $\widetilde{H}$, given by (2), are related by a first order differential operator $A$, given by (1), if and only if there exist a constant $\epsilon$ and a function $W$ such that the pair of Riccati equations (3) and (4) are satisfied simultaneously. Moreover, this means that both Hamiltonians can be factorized as

$$
H=A^{\dagger} A+\epsilon, \quad \widetilde{H}=A A^{\dagger}+\epsilon .
$$

Adding and subtracting equations (3) and (4) we obtain the equivalent pair which relates $V$ and $\widetilde{V}$

$$
\begin{aligned}
\widetilde{V}-\epsilon & =-(V-\epsilon)+2 W^{2}, \\
\widetilde{V} & =V+2 W^{\prime} .
\end{aligned}
$$


The function $W$ satisfying these equations is usually called superpotential, the constant $\epsilon$ is the factorization energy or factorization constant and $\widetilde{V}$ and $V$ (resp. $\widetilde{H}$ and $H$ ) are said to be partner potentials (resp. Hamiltonians).

Notice that the initial solvable Hamiltonian can indistinctly be chosen as $H$ or $\widetilde{H}$. In both cases the point will be to find a solution $W$ of the corresponding Riccati equation (3) or (4) for a specific factorization energy $\epsilon$. From this solution the expression for the (possibly) new potential follows immediately from (7).

\section{DILATION SYMMETRY AND REDUCTION OF A LINEAR SECOND-ORDER DIFFERENTIAL EQUATION}

Let us briefly recall a well-known method of relating a homogeneous linear second-order differential equation to a Riccati equation, which can be regarded as an application of the classical Lie theory of infinitesimal symmetries of differential equations. Its importance will become clear when applying the method to time-independent Schrödinger equations.

The homogeneous linear second-order differential equation

$$
\frac{d^{2} z}{d x^{2}}+b(x) \frac{d z}{d x}+c(x) z=0
$$

admits as an infinitesimal symmetry the vector field $X=z \partial / \partial z$ generating dilations (see e.g. [29]) in the variable $z$, which is defined for $z \neq 0$. According to the Lie theory of infinitesimal symmetries of differential equations, we should change the coordinate $z$ to a new one, $u=\varphi(z)$, such that the vector field $X=z \partial / \partial z$ becomes a translation generator $X=\partial / \partial u$ in the new variable. This change is determined by the equation $X u=1$, which leads to $u=\log |z|$, i.e. $|z|=e^{u}$. In both cases of regions with $z>0$ or $z<0$ we have

$$
\frac{d z}{d x}=z \frac{d u}{d x}, \quad \text { and } \quad \frac{d^{2} z}{d x^{2}}=z\left(\frac{d u}{d x}\right)^{2}+z \frac{d^{2} u}{d x^{2}},
$$

so the equation (8) becomes

$$
\frac{d^{2} u}{d x^{2}}+b(x) \frac{d u}{d x}+\left(\frac{d u}{d x}\right)^{2}+c(x)=0 .
$$

As the unknown function $u$ does not appear in the preceding equation but just its derivative, we can lower the order by introducing the new variable $w=d u / d x$. We arrive to the following Riccati equation for $w$

$$
\frac{d w}{d x}=-w^{2}-b(x) w-c(x) .
$$


Notice that from $d z / d x=z d u / d x$ and the definition of $w$ we have

$$
w=\frac{1}{z} \frac{d z}{d x} .
$$

The second order differential equation (8) is equivalent to the set of (9) and (10), because given a function $w$ satisfying (9), the function $z$ defined (up to a factor) by (10), i.e. $z(x)=\exp \left(\int^{x} w(\zeta) d \zeta\right.$ ), satisfies (8). We could have followed a similar pattern straightening out the vector field in the opposite sense, that is, by imposing $X u=-1$. This would have lead to $u=-\log |z|$, or $|z|=e^{-u}$. Now, in either case of $z>0$ or $z<0$ we have $d z / d x=-z d u / d x$ and $d^{2} z / d x^{2}=z(d u / d x)^{2}-z d^{2} u / d x^{2}$, so we finally obtain the Riccati equation

$$
\frac{d w}{d x}=w^{2}-b(x) w+c(x)
$$

where now

$$
w=\frac{d u}{d x}=-\frac{1}{z} \frac{d z}{d x}
$$

We will distinguish in what follows between these two alternatives of reduction of (8) by means of a subscript + or - in the corresponding functions, respectively. We remark that both are defined locally, that is, in open intervals where $z$ has a constant sign.

Let us apply these ideas to the particular case of the one-dimensional time-independent Schrödinger equation

$$
-\frac{d^{2} \phi}{d x^{2}}+(V(x)-\epsilon) \phi=0,
$$

where $V(x)$ is the potential and $\epsilon$ is some specific energy eigenvalue. As explained before, we can reduce (13) either to the pair

$$
W_{+}^{\prime}=-W_{+}^{2}+(V(x)-\epsilon), \quad W_{+}=\frac{1}{\phi} \frac{d \phi}{d x},
$$

or, alternatively, to the pair

$$
W_{-}^{\prime}=W_{-}^{2}-(V(x)-\epsilon), \quad W_{-}=-\frac{1}{\phi} \frac{d \phi}{d x} .
$$

The Riccati equations appearing in these pairs resemble those appearing in Section 1, namely equations (3) and (4), but in the systems (14) and (15) 
the unknown functions $W_{+}$and $W_{-}$are related by $W_{+}=-W_{-}$, while in both (3) and (4) the unknown $W$ is the same function.

However, the previous remark will be useful in the interpretation of equations (3) and (4). We can rewrite them as

$$
\begin{aligned}
& W^{\prime}=W^{2}-(V(x)-\epsilon), \\
& W^{\prime}=-W^{2}+(\widetilde{V}(x)-\epsilon) .
\end{aligned}
$$

Then, we can regard equation (16) (resp. equation (17)) as coming from a Schrödinger-type equation like (13) (resp. like $-d^{2} \widetilde{\phi} / d x^{2}+(\widetilde{V}(x)-\epsilon) \widetilde{\phi}=0$ ) by means of, respectively, the changes

$$
W=-\frac{1}{\phi} \frac{d \phi}{d x}, \quad \text { or } \quad W=\frac{1}{\widetilde{\phi}} \frac{d \widetilde{\phi}}{d x},
$$

so the two "eigenfunctions" $\phi$ and $\widetilde{\phi}$ of the mentioned Schrödinger-type equations are related by $\phi \widetilde{\phi}=$ Const. Of course, the changes (18) are defined locally, i.e. in common open intervals of the domains of $\phi$ and $\widetilde{\phi}$ determined by two consecutive zeros of $\phi$ or $\widetilde{\phi}$, or maybe by a zero and a boundary of the domain of the problem. Note that there is no reason why they should provide functions $\phi, \widetilde{\phi}$ defined in the same way in the entire domain of $W$, but in general they will be defined interval-wise. Moreover, if we choose the function $W$ of the two operators $A$ and $A^{\dagger}$ defined in (1) as given by (18) it holds $A \phi=0$ and $A^{\dagger} \widetilde{\phi}=0$.

We have seen that the Riccati equations (16) and (17) correspond by means of the changes (18) to two Schrödinger-type equations which in turn are equivalent to

$$
H \phi=\epsilon \phi, \quad \widetilde{H} \widetilde{\phi}=\epsilon \widetilde{\phi},
$$

where $H$ and $\widetilde{H}$ are given by (2). Then, it is equivalent to say that $H$ and $\widetilde{H}$ are $A$-related, with associated constant $\epsilon$, to say that the pair of functions $\phi$ and $\widetilde{\phi}$, which satisfy $\phi \widetilde{\phi}=$ Const., are respective eigenfunctions with eigenvalue $\epsilon$ of the Hamiltonians $H$ and $\widetilde{H}$. Each of these facts imply that both Hamiltonians can be factorized as in (5). We finally remark again that these factorizations make sense only locally, i.e. in common open intervals where $\phi$ and $\widetilde{\phi}$ are defined.

A special case where all becomes globally defined arises when $\phi$ or $\widetilde{\phi}$ is the ground state wave function of its respective Hamiltonian, having then no zeros in the entire domain of the problem. Then, remembering the equation (7) it is clear the relation of what we have just exposed with the Darboux transformations in the context of one-dimensional or Supersymmetric Quantum Mechanics, as it is shown, for example, in [7, pp. 7, $24]$. 
Moreover, for $\epsilon$ below the ground state energy of $H$ (resp. $\widetilde{H}$ ) it is sometimes possible to find a non-normalizable eigenfunction $\phi$ (resp. $\widetilde{\phi}$ ) of $H$ (resp. $\widetilde{H}$ ) without zeros, leading to physically interesting potentials $[4,5]$. However, as this procedure is slightly involved, we shall restrict for simplicity the discussions along this article to the previous case of $\epsilon$ equal to the ground state energy of $H$ or $\widetilde{H}$.

\section{TRANSFORMATION GROUP ON THE SET OF RICCATI EQUATIONS}

In this section we introduce the technique of relating different Riccati equations by means of a group action. The procedure, of key importance in what follows, was described in [27] in order to give a group theoretical explanation of some properties of the Riccati equation.

We recall that a general Riccati equation is the non-linear first order differential equation

$$
\frac{d y(x)}{d x}=a_{2}(x) y^{2}(x)+a_{1}(x) y(x)+a_{0}(x),
$$

where the coefficients $a_{i}(x), i=0,1,2$, are smooth functions of the independent variable $x$. A particular Riccati equation, determined by a specific election of these coefficient functions, can be regarded as a curve in $\mathbb{R}^{3}$, i.e., an element of $\operatorname{Map}\left(\mathbb{R}, \mathbb{R}^{3}\right)$.

We can transform every function $y(x)$ in the extended real line $\overline{\mathbb{R}}$ by an element of the group of smooth $S L(2, \mathbb{R})$-valued curves $\operatorname{Map}(\mathbb{R}, S L(2, \mathbb{R}))$, which from now on will be denoted $\mathcal{G}$, by means of the action $\Theta: \mathcal{G} \times$ $\operatorname{Map}(\mathbb{R}, \overline{\mathbb{R}}) \rightarrow \operatorname{Map}(\mathbb{R}, \overline{\mathbb{R}})$ defined as follows [27]:

$$
\begin{array}{r}
\Theta(A(x), y(x))=\frac{\alpha(x) y(x)+\beta(x)}{\gamma(x) y(x)+\delta(x)}, \quad \text { if } y(x) \neq-\frac{\delta(x)}{\gamma(x)}, \\
\Theta(A(x), \infty)=\frac{\alpha(x)}{\gamma(x)}, \quad \Theta\left(A(x),-\frac{\delta(x)}{\gamma(x)}\right)=\infty
\end{array}
$$

when

$$
A(x)=\left(\begin{array}{cc}
\alpha(x) & \beta(x) \\
\gamma(x) & \delta(x)
\end{array}\right) \in \mathcal{G}
$$

It is easy to check that the Riccati equation (20) transforms under the generic transformation $\bar{y}(x)=\Theta(A(x), y(x))$ into a new Riccati equation for $\bar{y}(x)$ with coefficients $\bar{a}_{i}(x), i=0,1,2$. The relation amongst the new 
and the old coefficients is given by

$$
\begin{aligned}
& \bar{a}_{2}=\delta^{2} a_{2}-\delta \gamma a_{1}+\gamma^{2} a_{0}+\gamma \delta^{\prime}-\delta \gamma^{\prime} \\
& \bar{a}_{1}=-2 \beta \delta a_{2}+(\alpha \delta+\beta \gamma) a_{1}-2 \alpha \gamma a_{0}+\delta \alpha^{\prime}-\alpha \delta^{\prime}+\beta \gamma^{\prime}-\gamma \beta^{\prime} \\
& \bar{a}_{0}=\beta^{2} a_{2}-\alpha \beta a_{1}+\alpha^{2} a_{0}+\alpha \beta^{\prime}-\beta \alpha^{\prime}
\end{aligned}
$$

where the prime denotes derivative respect to $x$. We would like to remark that similar transformations, using $G L(2, \mathbb{R})$ instead of $S L(2, \mathbb{R})$ have been used in [30, 31], also in connection with transformations of the Riccati equation. However, it seems that none of them noticed that these transformations constitute indeed a group action, as we will see next.

The previous relation can be written in a matrix form

$$
\begin{aligned}
\left(\begin{array}{c}
\bar{a}_{2} \\
\bar{a}_{1} \\
\bar{a}_{0}
\end{array}\right)= & \left(\begin{array}{ccc}
\delta^{2} & -\delta \gamma & \gamma^{2} \\
-2 \beta \delta & \alpha \delta+\beta \gamma & -2 \alpha \gamma \\
\beta^{2} & -\alpha \beta & \alpha^{2}
\end{array}\right)\left(\begin{array}{l}
a_{2} \\
a_{1} \\
a_{0}
\end{array}\right) \\
& +\left(\begin{array}{c}
\gamma \delta^{\prime}-\delta \gamma^{\prime} \\
\delta \alpha^{\prime}-\alpha \delta^{\prime}+\beta \gamma^{\prime}-\gamma \beta^{\prime} \\
\alpha \beta^{\prime}-\beta \alpha^{\prime}
\end{array}\right) .
\end{aligned}
$$

If we define the maps

$$
\begin{aligned}
B(A)= & \left(\begin{array}{ccc}
\delta^{2} & -\delta \gamma & \gamma^{2} \\
-2 \beta \delta & \alpha \delta+\beta \gamma & -2 \alpha \gamma \\
\beta^{2} & -\alpha \beta & \alpha^{2}
\end{array}\right), \\
\theta(A)= & \left.\begin{array}{c}
\gamma \delta^{\prime}-\delta \gamma^{\prime} \\
\delta \alpha^{\prime}-\alpha \delta^{\prime}+\beta \gamma^{\prime}-\gamma \beta^{\prime} \\
\alpha \beta^{\prime}-\beta \alpha^{\prime}
\end{array}\right),
\end{aligned}
$$

where $A \in \mathcal{G}$, it can be easily checked that $B$ is a linear representation of the group $\mathcal{G}$ on $\operatorname{Map}\left(\mathbb{R}, \mathbb{R}^{3}\right)$. The restriction of $B$ to the subgroup of constant $S L(2, \mathbb{R})$-valued curves is nothing but the adjoint action of $S L(2, \mathbb{R})$ on its Lie algebra [27]. On the other hand, it can be proved that $\theta$ defines an $1-$ cocycle for $B$, which means that (see [27, 32])

$$
\theta\left(A_{1} A_{2}\right)=B\left(A_{1}\right)\left(\theta\left(A_{2}\right)\right)+\theta\left(A_{1}\right), \quad \forall A_{1}, A_{2} \in \mathcal{G},
$$

and moreover $\theta$ is not an 1 -coboundary for $B$. Consequently, see e.g [32], the expression (27) defines an affine action of $\mathcal{G}$ on the set of general Riccati equations, which in turn can be identified with the set of curves on the Lie algebra $\mathfrak{s l}(2, \mathbb{R})$.

In practical terms, all this means that the composition of two transformations of type (27) on the set of Riccati equations is exactly the same as 
making only one transformation in the following way. If $T_{A}$ denotes the transformation of type (27) associated with $A \in \mathcal{G}$, then it holds

$$
T_{A_{1}} \circ T_{A_{2}}=T_{A_{1} A_{2}}, \quad \forall A_{1}, A_{2} \in \mathcal{G} .
$$

It is possible to generalize this affine action in more general situations, when an arbitrary finite dimensional Lie group is involved [33].

In short, if $y(x)$ is a solution of the equation (20) and we transform it by means of $\bar{y}(x)=\Theta(A(x), y(x))$, then $\bar{y}(x)$ will be a solution of the Riccati equation with coefficient functions (24), (25) and (26). By means of this technique we will prove the following result (the notation has been adapted to the one used along this article).

Theorem 4.1 (Finite difference Bäcklund algorithm $[4,28,34,35]$ ).

Let $w_{k}(x), w_{l}(x)$ be two solutions of the Riccati equations $w^{\prime}+w^{2}=$ $V(x)-\epsilon_{k}$ and $w^{\prime}+w^{2}=V(x)-\epsilon_{l}$, respectively, where $\epsilon_{k}<\epsilon_{l}$. Then the function $w_{k l}(x)$ defined by

$$
w_{k l}(x)=-w_{k}(x)-\frac{\epsilon_{k}-\epsilon_{l}}{w_{k}(x)-w_{l}(x)},
$$

is a solution of the Riccati equation $w^{\prime}+w^{2}=V(x)-2 w_{k}^{\prime}(x)-\epsilon_{l}$.

Proof. The function $w_{l}(x)$ satisfies the Riccati equation $w^{\prime}+w^{2}=$ $V(x)-\epsilon_{l}$ by hypothesis. We transform it by means of the element $A_{0}(x)$ of $\mathcal{G}$ given by

$$
A_{0}(x)=\frac{1}{\sqrt{a}}\left(\begin{array}{cc}
h(x) & -h^{2}(x)+a \\
-1 & h(x)
\end{array}\right)
$$

where $h(x)$ and $a$ are a function with the same domain as $w_{l}(x)$ and a positive constant, respectively. Notice that $A_{0}(x) \in \mathcal{G}$ since its determinant is always one, for all $x$ in the domain of $h(x)$. According to (21) and (22) we compute

$$
\Theta\left(A_{0}(x), w_{l}(x)\right)=\frac{h(x) w_{l}(x)-h^{2}(x)+a}{h(x)-w_{l}(x)}=-h(x)+\frac{a}{h(x)-w_{l}(x)} .
$$

This is a solution of the Riccati equation with coefficient functions given by (24), (25) and (26), with matrix elements $\alpha(x)=\delta(x)=h(x) / \sqrt{a}$, $\beta(x)=\left(-h^{2}(x)+a\right) / \sqrt{a}, \gamma(x)=-1 / \sqrt{a}$ and coefficients of the initial Riccati equation $a_{2}(x)=-1, a_{1}(x)=0$ and $a_{0}(x)=V(x)-\epsilon_{l}$. Simply 
performing the operations, we find

$$
\begin{aligned}
\bar{a}_{2}(x)= & \frac{1}{a}\left\{-h^{2}(x)-h^{\prime}(x)+V(x)-\epsilon_{l}+a\right\}-1, \\
\bar{a}_{1}(x)= & \frac{2 h(x)}{a}\left\{-h^{2}(x)-h^{\prime}(x)+V(x)-\epsilon_{l}+a\right\}, \\
\bar{a}_{0}(x)= & \frac{h^{2}(x)}{a}\left\{-h^{2}(x)-h^{\prime}(x)+V(x)-\epsilon_{l}+a\right\} \\
& +h^{2}(x)+h^{\prime}(x)-2 h^{\prime}(x)-a .
\end{aligned}
$$

Therefore, if the function $h(x)$ satisfies the Riccati equation $w^{2}+w^{\prime}=$ $V(x)-\epsilon_{k}$, with $\epsilon_{k}=\epsilon_{l}-a$, and we rename it as $h(x)=w_{k}(x)$, the new coefficients reduce to $\bar{a}_{2}(x)=-1, \bar{a}_{1}(x)=0$ and $\bar{a}_{0}(x)=V(x)-2 w_{k}^{\prime}(x)-$ $\epsilon_{l}$.

Let us note that in [4] the proof of the Theorem 4.1 was just sketched. In addition, there exists an alternative proof; see, e.g., Mielnik, Nieto and Rosas-Ortiz [28].

\section{GROUP ELEMENTS PRESERVING THE SUBSET OF RICCATI EQUATIONS $w^{\prime}+w^{2}=V(x)-\epsilon$}

We have just seen how the transformation group defined on the set of Riccati equations provides a direct proof of Theorem 4.1. It relates one solution $w_{l}(x)$ of the initial Riccati equation $w^{\prime}+w^{2}=V(x)-\epsilon_{l}$ with one solution $w_{k l}(x)$ of the final Riccati equation $w^{\prime}+w^{2}=V(x)-2 w_{k}^{\prime}(x)-$ $\epsilon_{l}$ by using a solution $w_{k}(x)$ of the intermediate Riccati equation $w^{\prime}+$ $w^{2}=V(x)-\epsilon_{k}$. These three Riccati equations can be obtained from another three Schrödinger-like equations by means of one of the reduction possibilities explained in Section 3. Moreover, those associated with the initial and intermediate Riccati equations, namely $-\psi^{\prime \prime}+\left(V(x)-\epsilon_{l}\right) \psi=$ 0 and $-\psi^{\prime \prime}+\left(V(x)-\epsilon_{k}\right) \psi=0$, can be seen as the arising eigenvalue equations for the two energies $\epsilon_{l}, \epsilon_{k}$ of the same potential $V(x)$, meanwhile the final Riccati equation can be associated to the eigenvalue equation for the potential $V(x)-2 w_{k}^{\prime}(x)$ with eigenvalue $\epsilon_{l}$.

Then, we are naturally led to the question of which are the most general elements of $\mathcal{G}$ preserving the subset of Riccati equations characterized by having the coefficients $a_{2}(x)=-1, a_{1}(x)=0$ and $a_{0}(x)$ equal to some function, which we will write as an expression of the form $V(x)-\epsilon$.

The Riccati equation we will start from is

$$
w^{\prime}=-w^{2}+V(x)-\epsilon,
$$


which according to (20) has the coefficients $a_{2}(x)=-1, a_{1}(x)=0$ and $a_{0}(x)=V(x)-\epsilon$. The condition for obtaining a final Riccati equation in the mentioned subset is

$$
\begin{aligned}
\left(\begin{array}{c}
-1 \\
0 \\
\bar{V}(x)-\bar{\epsilon}
\end{array}\right)= & \left(\begin{array}{ccc}
\delta^{2} & -\delta \gamma & \gamma^{2} \\
-2 \beta \delta & \alpha \delta+\beta \gamma & -2 \alpha \gamma \\
\beta^{2} & -\alpha \beta & \alpha^{2}
\end{array}\right)\left(\begin{array}{c}
-1 \\
0 \\
V(x)-\epsilon
\end{array}\right) \\
& +\left(\begin{array}{c}
\gamma \delta^{\prime}-\delta \gamma^{\prime} \\
\delta \alpha^{\prime}-\alpha \delta^{\prime}+\beta \gamma^{\prime}-\gamma \beta^{\prime} \\
\alpha \beta^{\prime}-\beta \alpha^{\prime}
\end{array}\right),
\end{aligned}
$$

for an $A(x) \in \mathcal{G}$ given by (23) to be determined, and where $\bar{V}(x)-\bar{\epsilon}$ will be in general different to $V(x)-\epsilon$. Therefore, the elements of the subset of $\mathcal{G}$ we are trying to characterize will not necessarily form a subgroup. The matrix equation (35) is equivalent to three scalar equations

$$
\begin{aligned}
-1 & =-\delta^{2}+\gamma^{2}(V(x)-\epsilon)+\gamma \delta^{\prime}-\delta \gamma^{\prime}, \\
0 & =2 \beta \delta-2 \alpha \gamma(V(x)-\epsilon)+\delta \alpha^{\prime}-\alpha \delta^{\prime}+\beta \gamma^{\prime}-\gamma \beta^{\prime}, \\
\bar{V}(x)-\bar{\epsilon} & =-\beta^{2}+\alpha^{2}(V(x)-\epsilon)+\alpha \beta^{\prime}-\beta \alpha^{\prime} .
\end{aligned}
$$

Differentiating $\operatorname{det} A(x)=\alpha(x) \delta(x)-\beta(x) \gamma(x)=1$ we have as well

$$
\alpha^{\prime} \delta+\delta^{\prime} \alpha-\gamma^{\prime} \beta-\beta^{\prime} \gamma=0
$$

Out of these four equations, (36), (37) and (39) will give conditions on the matrix elements $\alpha, \beta, \gamma, \delta$ and their derivatives such that the preserving condition be satisfied. The remaining (38) will define $\bar{V}(x)-\bar{\epsilon}$ in terms of all the other functions, including $V(x)-\epsilon$.

After taking the sum and the difference of (37) and (39) it follows

$$
\begin{aligned}
& (V(x)-\epsilon) \alpha^{2}=\frac{\alpha \beta \delta}{\gamma}+\frac{\delta \alpha \alpha^{\prime}}{\gamma}-\alpha \beta^{\prime} \\
& (V(x)-\epsilon) \gamma^{2}=\frac{\gamma \beta \delta}{\alpha}+\frac{\beta \gamma \gamma^{\prime}}{\alpha}-\gamma \delta^{\prime}
\end{aligned}
$$

Substituting them into (36) and (38) gives

$$
\begin{aligned}
-1 & =-\delta^{2}+\frac{\gamma \beta \delta}{\alpha}+\frac{\beta \gamma \gamma^{\prime}}{\alpha}-\delta \gamma^{\prime}, \\
\bar{V}(x)-\bar{\epsilon} & =-\beta^{2}+\frac{\alpha \beta \delta}{\gamma}+\frac{\delta \alpha \alpha^{\prime}}{\gamma}-\beta \alpha^{\prime} .
\end{aligned}
$$


Multiplying the first of these equations by $\alpha$ and the second by $\gamma$, and using the fact that $\alpha \delta-\beta \gamma=1$, we arrive to

$$
\begin{aligned}
\alpha & =\delta+\gamma^{\prime}, \\
(\bar{V}(x)-\bar{\epsilon}) \gamma & =\beta+\alpha^{\prime} .
\end{aligned}
$$

Substituting (42) into (41) yields

$$
(V(x)-\epsilon) \gamma=\beta-\delta^{\prime} .
$$

We have two relations amongst the functions $\alpha, \beta, \gamma$ and $\delta$, namely (42) and the determinant condition, so we can express these matrix elements in terms of only two of them and their derivatives. Then we have $\alpha=\delta+\gamma^{\prime}$ and $\beta=\left(\delta\left(\delta+\gamma^{\prime}\right)-1\right) / \gamma$. Using moreover the fact that

$$
\frac{\delta^{\prime}}{\gamma}=\left(\frac{\delta}{\gamma}\right)^{\prime}+\frac{\delta \gamma^{\prime}}{\gamma^{2}}
$$

the equation (44) becomes

$$
\left(-\frac{\delta}{\gamma}\right)^{\prime}+\left(-\frac{\delta}{\gamma}\right)^{2}=V(x)+\frac{1}{\gamma^{2}}-\epsilon
$$

so the new function $v$ defined as $v=-\delta / \gamma$ must satisfy the Riccati equation

$$
v^{\prime}+v^{2}=V(x)+\frac{1}{\gamma^{2}}-\epsilon .
$$

Now, substituting in (43) the expressions of $\beta$ and $\alpha^{\prime}$ in terms of $\delta, \gamma$ and their derivatives, using the definition of $v$ and the equation (45) gives

$$
\bar{V}(x)-\bar{\epsilon}=V(x)-2\left(\frac{\gamma^{\prime}}{\gamma} v+v^{\prime}\right)+\frac{\gamma^{\prime \prime}}{\gamma}-\epsilon .
$$

It only remains to find the expression of the function solution of the final Riccati equation, in terms of $w$ and $v$. The $S L(2, \mathbb{R})$-valued curve used for the transformation can be written as

$$
C_{0}(x)=\gamma\left(\begin{array}{cc}
-v+\frac{\gamma^{\prime}}{\gamma} & v^{2}-v \frac{\gamma^{\prime}}{\gamma}-\frac{1}{\gamma^{2}} \\
1 & -v
\end{array}\right),
$$

so the desired function is

$$
\begin{aligned}
\bar{w}(x) & =\Theta\left(C_{0}(x), w(x)\right)=\frac{-v w+w \gamma^{\prime} / \gamma-1 / \gamma^{2}+v^{2}-v \gamma^{\prime} / \gamma}{w-v} \\
& =-v-\frac{1 / \gamma^{2}}{w-v}+\frac{\gamma^{\prime}}{\gamma} .
\end{aligned}
$$


In short, we have just proved the following Theorem:

THEOREM 5.1. Let $w(x)$ be a solution of the Riccati equation

$$
w^{\prime}+w^{2}=V(x)-\epsilon
$$

for some function $V(x)$ and some constant $\epsilon$, and $\gamma(x)$ a never vanishing differentiable function defined on the domain of $V(x)$. If $v(x)$ is a solution of the Riccati equation

$$
v^{\prime}+v^{2}=V(x)+\frac{1}{\gamma^{2}(x)}-\epsilon,
$$

such that is defined in the same domain as $w(x)$ and $w(x)-v(x)$ does not vanish, then the function $\bar{w}(x)$ defined by

$$
\bar{w}(x)=-v(x)-\frac{1 / \gamma^{2}(x)}{w(x)-v(x)}+\frac{\gamma^{\prime}(x)}{\gamma(x)}
$$

is a solution of the Riccati equation

$$
\bar{w}^{\prime}+\bar{w}^{2}=V(x)-2\left(\frac{\gamma^{\prime}}{\gamma} v+v^{\prime}\right)+\frac{\gamma^{\prime \prime}}{\gamma}-\epsilon .
$$

Needless to say, the coefficients of the final equation can be calculated directly by using (24), (25), (26) and taking into account (46), (48) and (49).

Corollary 5.1. The Theorem 4.1 is a particular case of Theorem 5.1.

Proof. It is sufficient to choose in Theorem $5.1 w(x)=w_{l}(x), v(x)=$ $w_{k}(x), \epsilon=\epsilon_{l}$ and $\gamma=1 / \sqrt{\epsilon_{l}-\epsilon_{k}}$, with $\epsilon_{k}<\epsilon_{l}$.

Theorem 5.1 has a counterpart for linear second-order differential equations of Schrödinger type, which will be in turn of direct interest in physical applications. The key is to use in a inverse way the reduction procedure outlined in Section 3.

Consider the solution $w$ of the Riccati equation (48). We can define (locally and up to a non-vanishing multiplicative constant) the new variable $\phi_{w}$ as

$$
\phi_{w}(x)=\exp \left(\int^{x} w(\xi) d \xi\right)
$$


which will satisfy

$$
-\phi_{w}^{\prime \prime}+(V(x)-\epsilon) \phi_{w}=0,
$$

for the specific constant $\epsilon$. Analogously, by considering a solution $v$ of the Riccati equation (49) we can define (locally etc.) $\phi_{v}$ as

$$
\phi_{v}(x)=\exp \left(\int^{x} v(\xi) d \xi\right)
$$

which will satisfy

$$
-\phi_{v}^{\prime \prime}+\left(V(x)+\frac{1}{\gamma^{2}(x)}-\epsilon\right) \phi_{v}=0,
$$

for the same specific constant $\epsilon$. Then the function $\bar{w}$ defined by (50) will satisfy the Riccati equation (51). We could define as well (locally etc.) the new function $\phi_{\bar{w}}$ as

$$
\phi_{\bar{w}}(x)=\exp \left(\int^{x} \bar{w}(\xi) d \xi\right)
$$

which in turn will satisfy

$$
-\phi_{\bar{w}}^{\prime \prime}+\left\{V(x)-2\left(\frac{\gamma^{\prime}}{\gamma} v+v^{\prime}\right)+\frac{\gamma^{\prime \prime}}{\gamma}-\epsilon\right\} \phi_{\bar{w}}=0 .
$$

What has to be done now is to relate the function $\phi_{\bar{w}}$ with $\phi_{w}$ and $\phi_{v}$, taking into account the relation amongst $\bar{w}, w$ and $v$.

Proposition 5.1. Let $w, v, \bar{w}$ be the functions for which the Theorem 5.1 holds, and $\phi_{w}, \phi_{v}, \phi_{\bar{w}}$ the ones defined by (52), (53) and (54), respectively. Then we have

$$
\frac{\phi_{w}^{\prime}}{\phi_{w}}=w, \quad \frac{\phi_{v}^{\prime}}{\phi_{v}}=v, \quad \frac{\phi_{\bar{w}}^{\prime}}{\phi_{\bar{w}}}=\bar{w}
$$

and it holds

$$
\phi_{\bar{w}}=\gamma\left(-\frac{d}{d x}+\frac{\phi_{v}^{\prime}}{\phi_{v}}\right) \phi_{w},
$$

up to a non-vanishing multiplicative constant. 
Proof. The first assertion is immediate. As a consequence, we have $\gamma\left(-\frac{d}{d x}+\frac{\phi_{v}^{\prime}}{\phi_{v}}\right) \phi_{w}=\gamma(v-w) \phi_{w}$. Taking the logarithmic derivative

$$
\begin{aligned}
\frac{\left(\gamma(v-w) \phi_{w}\right)^{\prime}}{\gamma(v-w) \phi_{w}} & =\frac{\gamma^{\prime}}{\gamma}+\frac{v^{\prime}-w^{\prime}}{v-w}+\frac{\phi_{w}^{\prime}}{\phi_{w}} \\
& =\frac{\gamma^{\prime}}{\gamma}+\frac{w^{2}-v^{2}}{v-w}+\frac{1 / \gamma^{2}}{v-w}+w=\frac{\gamma^{\prime}}{\gamma}-w-v+\frac{1 / \gamma^{2}}{v-w}+w \\
& =\frac{\gamma^{\prime}}{\gamma}-v+\frac{1 / \gamma^{2}}{v-w}=\bar{w}=\frac{\phi_{\bar{w}}^{\prime}}{\phi_{\bar{w}}}
\end{aligned}
$$

where it has been used the equations (48), (49) and (50).

With the previous results we have the following:

TheOREM 5.2. Let $\phi_{w}(x)$ be a solution of the homogeneous linear second order differential equation

$$
-\phi_{w}^{\prime \prime}+(V(x)-\epsilon) \phi_{w}=0,
$$

for some specific function $V(x)$ and constant $\epsilon$, and $\gamma(x)$ a never vanishing differentiable function defined on the domain of $V(x)$. If the function $\phi_{v}(x) \neq \phi_{w}(x)$ is a solution of the equation

$$
-\phi_{v}^{\prime \prime}+\left(V(x)+\frac{1}{\gamma^{2}(x)}-\epsilon\right) \phi_{v}=0
$$

defined in the same domain as $\phi_{w}(x)$, then the function $\phi_{\bar{w}}(x)$ defined (up to a non-vanishing multiplicative constant) by

$$
\phi_{\bar{w}}=\gamma\left(-\frac{d}{d x}+\frac{\phi_{v}^{\prime}}{\phi_{v}}\right) \phi_{w}
$$

satisfies the new equation

$$
-\phi_{\bar{w}}^{\prime \prime}+\left\{V(x)-2\left(\frac{\gamma^{\prime}}{\gamma} v+v^{\prime}\right)+\frac{\gamma^{\prime \prime}}{\gamma}-\epsilon\right\} \phi_{\bar{w}}=0
$$

where the function $v(x)$ is defined (locally) as $\phi_{v}^{\prime} / \phi_{v}=v$.

Note that Theorems 5.1 and 5.2 are invariant under the change of sign of $\gamma$. 


\section{FINITE DIFFERENCE ALGORITHM AND INTERTWINED HAMILTONIANS FROM A GROUP THEORETICAL VIEWPOINT}

We have already said that the finite difference algorithm, based on the Theorem 4.1, appeared in [4] when the authors wanted to iterate the standard first order intertwining technique. This idea has been kept also in subsequent works [5, 19], and in all of these articles the algorithm has been shown to be of use for obtaining new exactly solvable Hamiltonians. Moreover, the proof of Theorem 4.1 given recently by Mielnik, Nieto and Rosas-Ortiz, alternative to that which has been given here, still relies on the idea of iteration of the intertwining technique, see [28, Sec. 2] for details.

On the other hand, we have given a direct proof of Theorem 4.1 by making use of the action of $\mathcal{G}$ on the set of Riccati equations, and we wonder whether it is possible to establish a further relation between this transformation group and the (maybe iterated) intertwining technique.

The important result, which we show next, is the following. By using properly the finite difference algorithm just once, jointly with the reduction procedure described in Section 3, it is possible to explain from a group theoretical viewpoint the usual problem of $A$-related or intertwined Hamiltonians.

With this aim, let us consider two Hamiltonians

$$
H_{0}=-\frac{d^{2}}{d x^{2}}+V_{0}(x), \quad H_{1}=-\frac{d^{2}}{d x^{2}}+V_{1}(x),
$$

which by hypothesis are $A_{1}$-related, i.e. $A_{1} H_{1}=H_{0} A_{1}$ and $H_{1} A_{1}^{\dagger}=A_{1}^{\dagger} H_{0}$, where

$$
A_{1}=\frac{d}{d x}+w_{1}, \quad A_{1}^{\dagger}=-\frac{d}{d x}+w_{1},
$$

and $w_{1}$ is a function to be determined.

Assume that $H_{0}$ is an exactly solvable Hamiltonian for which we know a complete set of square-integrable eigenfunctions $\psi_{n}^{(0)}$ with respective energies $E_{n}, n=0,1,2, \ldots$ We have seen in Section 3 that, in particular,

$$
\begin{aligned}
& V_{0}(x)-E_{0}=w_{1}^{2}\left(x, E_{0}\right)+w_{1}^{\prime}\left(x, E_{0}\right), \\
& V_{1}(x)-E_{0}=w_{1}^{2}\left(x, E_{0}\right)-w_{1}^{\prime}\left(x, E_{0}\right),
\end{aligned}
$$

or equivalently

$$
\begin{aligned}
V_{0}(x)-E_{0} & =-\left(V_{1}(x)-E_{0}\right)+2 w_{1}^{2}\left(x, E_{0}\right) \\
V_{1}(x) & =V_{0}(x)-2 w_{1}^{\prime}\left(x, E_{0}\right)
\end{aligned}
$$


where we have chosen $w_{1}\left(x, E_{0}\right)$ as

$$
w_{1}\left(x, E_{0}\right)=\psi_{0}^{(0) \prime} / \psi_{0}^{(0)}
$$

Up to a non-vanishing multiplicative constant, we define the function $\psi_{0}^{(1)}$ as $\psi_{0}^{(1)}=1 / \psi_{0}^{(0)}$. We have as well

$$
w_{1}\left(x, E_{0}\right)=-\psi_{0}^{(1) \prime} / \psi_{0}^{(1)} .
$$

Then, both Hamiltonians factorize as

$$
H_{0}=A_{1}\left(E_{0}\right) A_{1}^{\dagger}\left(E_{0}\right)+E_{0}, \quad H_{1}=A_{1}^{\dagger}\left(E_{0}\right) A_{1}\left(E_{0}\right)+E_{0} .
$$

We have made explicit $E_{0}$ in the function $w_{1}$ and, as a consequence, in the operators $A_{1}$ and $A_{1}^{\dagger}$. However, it should be considered as a label reminding the factorization we are working with rather than as a functional dependence. From (67) and (68) we have $A_{1}^{\dagger}\left(E_{0}\right) \psi_{0}^{(0)}=0$ and $A_{1}\left(E_{0}\right) \psi_{0}^{(1)}=0$; as a result $H_{1} \psi_{0}^{(1)}=E_{0} \psi_{0}^{(1)}$ and $H_{0} \psi_{0}^{(0)}=E_{0} \psi_{0}^{(0)}$. As $\psi_{0}^{(0)}$ has no zeros in the domain of $V_{0}(x)$, all the functions defined in this Section will be globally defined provided that such a domain is connected.

The equation (66) relates the new potential $V_{1}(x)$ and the old one $V_{0}(x)$. As it is well known, due to the $A_{1}\left(E_{0}\right)$-relationship of the Hamiltonians $H_{0}$ and $H_{1}$, the normalized eigenfunctions of $H_{1}$ can be obtained transforming those of $H_{0}$ by means of the operator $A_{1}^{\dagger}\left(E_{0}\right)$ except $\psi_{0}^{(0)}$, since $A_{1}^{\dagger}\left(E_{0}\right) \psi_{0}^{(0)}=0$. In fact, a simple calculation shows that the functions

$$
\psi_{n}^{(1)}=\frac{A_{1}^{\dagger}\left(E_{0}\right) \psi_{n}^{(0)}}{\sqrt{E_{n}-E_{0}}},
$$

satisfy

$$
H_{1} \psi_{n}^{(1)}=E_{n} \psi_{n}^{(1)} \quad \text { and } \quad\left(\psi_{n}^{(1)}, \psi_{m}^{(1)}\right)=\delta_{n m},
$$

for all $n, m=1,2,3, \ldots$, provided that the functions $\psi_{n}^{(0)}$ are normalized.

Although the function $\psi_{0}^{(1)}$ satisfies $H_{1} \psi_{0}^{(1)}=E_{0} \psi_{0}^{(1)}$, it does not correspond to a physical state of $H_{1}$ since it is not normalizable, which means that $E_{0}$ will not belong to the spectrum of $H_{1}$. For this reason the Hamiltonians $H_{1}$ and $H_{0}$ are said to be quasi-isospectral.

Let us formulate now these results in terms of the transformation group on the set of Riccati equations introduced in Section 4. By hypothesis we have

$$
H_{0} \psi_{n}^{(0)}=E_{n} \psi_{n}^{(0)}, \quad n=0,1,2, \ldots
$$


As $H_{0}$ is given by (61), the spectral equation (72) can be written as the following set of equations

$$
-\psi_{n}^{(0) \prime \prime}+\left(V_{0}(x)-E_{n}\right) \psi_{n}^{(0)}=0, \quad n=0,1,2, \ldots
$$

We introduce the new functions

$$
w_{1}\left(E_{n}\right)=\frac{\psi_{n}^{(0) \prime}}{\psi_{n}^{(0)}}, \quad n=0,1,2, \ldots,
$$

where the dependence on $x$ has been omitted for brevity. As we know from Section 3, these transformations will be defined locally, i.e., for each $n$ the domain of $w_{1}\left(E_{n}\right)$ will be the union of the open intervals contained between two consecutive zeros of $\psi_{n}^{(0)}$ or maybe a zero and one boundary of the domain of $V_{0}(x)$. In particular, $w_{1}\left(E_{0}\right)$ is defined globally in the entire domain of $V_{0}(x)$ as $\psi_{0}^{(0)}$ have no zeros there. Therefore, the set of equations (73) reads in the new variables as the set

$$
w_{1}^{\prime}\left(E_{n}\right)+w_{1}^{2}\left(E_{n}\right)=V_{0}(x)-E_{n}, \quad n=0,1,2, \ldots,
$$

that is, the functions $w_{1}\left(E_{n}\right)$ are respective solutions of the Riccati equations

$$
w^{\prime}+w^{2}=V_{0}(x)-E_{n}, \quad n=0,1,2, \ldots .
$$

Let us apply the Theorem 4.1 to this situation. We act on the set of all equations (75) but one by means of suitable group elements of $\mathcal{G}$. These $S L(2, \mathbb{R})$-valued curves are constructed by means of the solution of the equation of the set (75) which is to be set aside. In order to avoid singularities, this solution should be the one with $n=0$.

The mentioned elements of $\mathcal{G}$ are analogous to the one used in the proof of Theorem 4.1. They turn out to be

$$
B_{n}=\frac{1}{\sqrt{E_{n}-E_{0}}}\left(\begin{array}{cc}
w_{1}\left(E_{0}\right) & -w_{1}^{2}\left(E_{0}\right)+E_{n}-E_{0} \\
-1 & w_{1}\left(E_{0}\right)
\end{array}\right), \quad n=1,2, \ldots .
$$

We define the new functions $\bar{w}_{1}\left(E_{n}\right)$ by

$$
\begin{aligned}
\bar{w}_{1}\left(E_{n}\right) & =\Theta\left(B_{n}, w_{1}\left(E_{n}\right)\right)=\frac{w_{1}\left(E_{0}\right) w_{1}\left(E_{n}\right)-w_{1}^{2}\left(E_{0}\right)+E_{n}-E_{0}}{w_{1}\left(E_{0}\right)-w_{1}\left(E_{n}\right)} \\
& =-w_{1}\left(E_{0}\right)-\frac{E_{0}-E_{n}}{w_{1}\left(E_{0}\right)-w_{1}\left(E_{n}\right)}, \quad n=1,2, \ldots
\end{aligned}
$$

By Theorem 4.1 these functions satisfy, respectively, the new Riccati equations

$$
\bar{w}^{\prime}+\bar{w}^{2}=V_{1}(x)-E_{n}, \quad n=1,2, \ldots,
$$


where $V_{1}(x)=V_{0}(x)-2 w_{1}^{\prime}\left(E_{0}\right)$. We can define (locally etc.) the new set of functions $\phi_{n}^{(1)}$, for $n=1,2, \ldots$, by

$$
\phi_{n}^{(1)}(x)=\exp \left(\int^{x} \bar{w}_{1}\left(\xi, E_{n}\right) d \xi\right), \quad n=1,2, \ldots,
$$

which therefore satisfy, respectively, a linear second-order differential equation of the set

$$
-\phi^{\prime \prime}+\left(V_{1}(x)-E_{n}\right) \phi=0, \quad n=1,2, \ldots .
$$

Then, $\phi_{n}^{(1)}$ are eigenfunctions of the Hamiltonian $H_{1}=-\frac{d^{2}}{d x^{2}}+V_{1}(x)$ with associated eigenvalues $E_{n}$, for $n=1,2, \ldots$ As a consequence, they can be written as the linear combinations $\phi_{n}^{(1)}=\psi_{n}^{(1)}+\lambda_{n} \psi_{n}^{(1)}(x) \int^{x} \frac{d \xi}{\psi_{n}^{(1) 2}(\xi)}$, for all $n=1,2, \ldots$, up to non-vanishing constant factors, where $\lambda_{n}$ are still unknown constants and it has been used the well-known Liouville formula for finding the second linearly independent solution of each equation of (80) starting from $\psi_{n}^{(1)}$.

Now, the important point is that each of the functions $\phi_{n}^{(1)}$ turns out to be the same as $\psi_{n}^{(1)}$, up to a non-vanishing constant factor, i.e. the previous constants $\lambda_{n}$ are all zero. In fact, the logarithmic derivative of $\psi_{n}^{(1)}$ is

$$
\begin{aligned}
\frac{\psi_{n}^{(1) \prime}}{\psi_{n}^{(1)}}= & \frac{\frac{d}{d x}\left(A_{1}^{\dagger}\left(E_{0}\right) \psi_{n}^{(0)}\right)}{A_{1}^{\dagger}\left(E_{0}\right) \psi_{n}^{(0)}}=\frac{\frac{d}{d x}\left(-\psi_{n}^{(0) \prime}+\frac{\psi_{0}^{(0) \prime}}{\psi_{0}^{(0)}} \psi_{n}^{(0)}\right)}{-\psi_{n}^{(0) \prime}+\frac{\psi_{0}^{(0) \prime}}{\psi_{0}^{(0)}} \psi_{n}^{(0)}} \\
= & \frac{-\psi_{n}^{(0) \prime \prime}+\frac{\psi_{0}^{(0) \prime \prime}}{\psi_{0}^{(0)}} \psi_{n}^{(0)}-\left(\frac{\psi_{0}^{(0) \prime}}{\psi_{0}^{(0)}}\right)^{2} \psi_{n}^{(0)}+\frac{\psi_{0}^{(0) \prime}}{\psi_{0}^{(0)}} \psi_{n}^{(0) \prime}}{-\psi_{n}^{(0) \prime}+\frac{\psi_{0}^{(0) \prime}}{\psi_{0}^{(0)}} \psi_{n}^{(0)}} .
\end{aligned}
$$

Taking common factor $\psi_{n}^{(0)}$ in both numerator and denominator, using the relations

$$
\frac{\psi_{n}^{(0) \prime \prime}}{\psi_{n}^{(0)}}=V_{0}(x)-E_{n}, \quad n=0,1,2, \ldots,
$$

and the definitions (74), we arrive to

$$
\frac{\psi_{n}^{(1) \prime}}{\psi_{n}^{(1)}}=-w_{1}\left(E_{0}\right)-\frac{E_{0}-E_{n}}{w_{1}\left(E_{0}\right)-w_{1}\left(E_{n}\right)}=\bar{w}_{1}\left(E_{n}\right)=\frac{\phi_{n}^{(1) \prime}}{\phi_{n}^{(1)}},
$$

for $n=1,2, \ldots$, therefore $\psi_{n}^{(1)}$ and $\phi_{n}^{(1)}$ must be proportional. It is also clear that these equations hold interval-wise. 
Now, as far as $\psi_{0}^{(0)}$ is concerned, it is clear that Theorem 4.1 would make no sense for $w_{k}(x)=w_{l}(x)$ and $\epsilon_{k}=\epsilon_{l}$. In a similar way, we cannot put $E_{n}=E_{0}$ in $(76)$ : the normalizing factors $1 / \sqrt{E_{n}-E_{0}}$, which were introduced in order to get $S L(2, \mathbb{R})$-valued curves, would no longer make sense because these matrices, after dropping out such factors would have zero determinant. This means that one cannot use a transformation of type (77) with $E_{n}=E_{0}$ for the function $w_{1}\left(E_{0}\right)$ itself. However, the associated function to $\psi_{0}^{(1)}$ at the Riccati level is just given by (68), i.e. the new function $\bar{w}_{1}\left(E_{0}\right)=-w_{1}\left(E_{0}\right)$ satisfies an equation of type (78) for $n=0$, which is precisely (64). In short, the equation $A_{1}^{\dagger}\left(E_{0}\right) \psi_{0}^{(0)}=0$ is translated at the Riccati level into the fact that $w_{1}\left(E_{0}\right)$ cannot be transformed in the sense mentioned above. Conversely, it is not possible to write $\bar{w}_{1}\left(E_{0}\right)=$ $\Theta\left(B_{0}, w_{1}\left(E_{0}\right)\right)$ for $B_{0} \in \mathcal{G}$.

We have just explained the problem of two $A_{1}\left(E_{0}\right)$-related Hamiltonians by means of Theorem 4.1, which in turn is a particular case of Theorem 5.1. For the sake of completeness, let us show briefly how Theorem 5.2 applies to the same problem. Consider the set of equations (73), where we retain again the one with $n=0$, which will play the rôle of equation (58). All the others will play the rôle of equation (57). For each $n=1,2, \ldots$, the function $\gamma$ must be defined by

$$
-E_{0}=-E_{n}+\frac{1}{\gamma^{2}}
$$

Thus, we can choose $\gamma=1 / \sqrt{E_{n}-E_{0}}$. According to (59) and (60) the functions

$$
\varphi_{n}^{(1)}=\frac{1}{\sqrt{E_{n}-E_{0}}}\left(-\frac{d}{d x}+\frac{\psi_{0}^{(0) \prime}}{\psi_{0}^{(0)}}\right) \psi_{n}^{(0)}, \quad n=1,2, \ldots
$$

satisfy, respectively,

$$
-\varphi^{\prime \prime}+\left(V_{0}(x)-2 w_{1}^{\prime}\left(E_{0}\right)-E_{n}\right) \varphi=0, \quad n=1,2, \ldots,
$$

where it has been used $w_{1}\left(E_{0}\right)=\psi_{0}^{(0) \prime} / \psi_{0}^{(0)}$. In this way we have recovered the normalized eigenfunctions (70) associated to the new potential $V_{1}(x)=$ $V_{0}(x)-2 w_{1}^{\prime}\left(x, E_{0}\right)$.

\section{ILLUSTRATIVE EXAMPLES}

In this Section we will apply Theorem 5.2 to some cases where $\gamma(x)$ is not a constant, what provides a more general situation than the usual intertwining technique. However, note that with this method we will be able to find 
potentials for which one eigenfunction and its corresponding eigenvalue are exactly known. We will use a slight generalization of two well-known types of potentials, namely the radial oscillator and Coulomb potentials, which consists of taking the most general intervals of the appearing parameters such that it is possible to find square-integrable eigenfunctions.

\subsection{Radial oscillator-like potentials}

Let us consider the family of potentials

$$
V_{l, b}(x)=\frac{b^{2} x^{2}}{4}+\frac{l(l+1)}{x^{2}}
$$

where $x \in(0, \infty)$ and $l, b$ are real parameters. Each member can be regarded as being part of a pair of shape invariant partner potentials, with associated transformation law $l \rightarrow l+1$ for the parameter $l[1,9]$. This fact allows to find the eigenvalues and the corresponding eigenfunctions, even normalized, in an algebraic way. The key is to find functions in the kernel of the first order differential operator $d / d x-(l+1) / x+b x / 2$ which be normalizable with respect to the norm induced by the standard scalar product defined in $L^{2}(0, \infty)$. That will provide the ground state eigenfunction. The eigenfunctions of the excited states are obtained from the iterated action of $-d / d x-(l+1) / x+b x / 2$, with appropriate $l$ at each step, times some suitable factors, on the ground state eigenfunction. However, the point is that with this procedure, one obtains the boundary conditions of the eigenfunctions as a consequence rather than being a priori requirements. The result for this family of potentials are the normalized eigenfunctions (up to a modulus one factor) [36]

$$
\zeta_{k}^{l, b}(x)=\sqrt{\frac{\Gamma(k+1)}{\Gamma(k+l+3 / 2)}}\left(\frac{b^{2 l+3}}{2^{2 l+1}}\right)^{1 / 4} x^{l+1} e^{-b x^{2} / 4} L_{k}^{l+1 / 2}\left(\frac{b x^{2}}{2}\right),
$$

where $k=0,1,2, \ldots$, for $l>-3 / 2$ and $b>0$. The notation $L_{n}^{a}(u)$ means the Laguerre polynomial of degree $n$ and parameter $a$ in the variable $u$.

Note that in the interval $l \in(-3 / 2,-1)$ these eigenfunctions go to infinity as $x$ tends to zero, contrary to the usual requirement of going to zero, in spite of the fact of being square-integrable.

The problem of the quantum-mechanical motion of a particle in a potential on the half line $(0, \infty)$ has been carefully studied in [37]. It has been shown there that the operator $H=-\frac{d^{2}}{d x^{2}}+V(x)$, with domain $C_{0}^{\infty}(0, \infty)$ of differentiable functions with compact support in $(0, \infty), V$ being a continuous real-valued function on $(0, \infty)$, is a symmetric operator and that it is essentially self-adjoint if and only if $V(x)$ is in the limit point case in both zero and infinity [37, Theorem X.7]. In the case we have in hand, what 
happens is that the potentials of the family (83) lead to essentially selfadjoint Hamiltonians $-d^{2} / d x^{2}+V_{l, b}(x)$ for the range $b>0$ and $l>-3 / 2$, with different self-adjoint extensions in each of the intervals $l \in(-3 / 2,-1)$ and $l \in(-1, \infty)$, the first including eigenfunctions which do not necessarily go to zero as $x \rightarrow 0$. We will see that one eigenfunction arising when $l \in(-3 / 2,-1)$ provides an interesting application, for the family of potentials (83), of our new method generalizing the first order intertwining technique.

In both cases, the corresponding eigenvalues to the eigenfunctions (84) for the potentials (83) are [36]:

$$
E_{k}^{l, b}=b\left(2 k+l+\frac{3}{2}\right), \quad k=0,1,2, \ldots
$$

If $b=2$ these eigenvalues reduce to those of [38]. Compare also with the eigenfunctions and eigenvalues given in [15, pp. 391-2].

EXAMPLE 7.1. Let us consider the following variant of the family of potentials (83)

$$
V_{l, b}(x)=\frac{b^{2} x^{2}}{4}+\frac{l(l+1)}{x^{2}}-b\left(l+\frac{3}{2}\right),
$$

where $x \in(0, \infty), l>-3 / 2$ and $b>0$. The normalized eigenfunctions are given again by (84), with the same peculiarities, but the corresponding eigenvalues are now:

$$
E_{k}^{l, b}=2 b k, \quad k=0,1,2, \ldots
$$

We would like to apply Theorem 5.2 , by using two potentials of the family (86) for different specific values of $l$. The difference between them should be a positive function in $(0, \infty)$ in order to define appropriately $\gamma(x)$ as required by the Theorem. We have

$$
V_{l, b}(x)-V_{l+r, b}(x)=r\left(b-\frac{2 l+1+r}{x^{2}}\right),
$$

where $r$ is a positive integer. Since $b>0$, the condition for the right hand side to be always positive is that $2 l+1+r<0$. We can find a solution if $r=1$, since then it should happen $2 l+2<0$ or equivalently $l<-1$. For $r=2,3, \ldots$, we would find $l<-3 / 2$, which is incompatible with the range of $l$. Then, we have to choose $r=1,-3 / 2<l<-1$ and therefore an appropriate function $\gamma(x)$ is

$$
\gamma_{l, b}(x)=\left(b-\frac{2 l+2}{x^{2}}\right)^{-1 / 2} .
$$


We will transform an eigenstate of $V_{l+1, b}(x)$ by making use of the eigenstate of $V_{l, b}(x)$ with the same energy, i.e. with the same $k$. Consider the functions

$$
v_{k}^{l, b}(x)=\frac{1}{\zeta_{k}^{l, b}(x)} \frac{d \zeta_{k}^{l, b}(x)}{d x},
$$

one of which will be used to find the final potential according to (60). Due to the presence of the Laguerre polynomials in $(84), \zeta_{k}^{l, b}(x)$ has $k$ zeros in $(0, \infty)$ and therefore $v_{k}^{l, b}(x)$, as well as the final potential, have $k$ singularities in the same interval. In order to avoid them, we choose $k=0$. Summarizing, we transform the eigenfunction $\zeta_{0}^{l+1, b}(x)$ obeying

$$
-\frac{d^{2} \zeta_{0}^{l+1, b}(x)}{d x^{2}}+V_{l+1, b}(x) \zeta_{0}^{l+1, b}(x)=0,
$$

by means of the solution $\zeta_{0}^{l, b}(x)$ of a equation similar to (88) but with $l$ instead of $l+1$. Since $l \in(-3 / 2,-1)$, both of the original eigenfunction $\zeta_{0}^{l+1, b}(x)$ and the intermediate one $\zeta_{0}^{l, b}(x)$ are square-integrable, but this last goes to infinity when $x \rightarrow 0$.

After some calculations, the image potential becomes

$$
\begin{aligned}
V_{l, b}^{i m}(x) & =V_{l+1, b}(x)-2\left(\frac{\gamma_{l, b}^{\prime}}{\gamma_{l, b}} v_{0}^{l, b}+\frac{d v_{0}^{l, b}}{d x}\right)+\frac{\gamma_{l, b}^{\prime \prime}}{\gamma_{l, b}} \\
& =\frac{b^{2} x^{2}}{4}+\frac{(l+1)(l+2)}{x^{2}}-b\left(l+\frac{3}{2}\right)+\frac{6 b(l+1)}{\left(b x^{2}-2(l+1)\right)^{2}},
\end{aligned}
$$

for which we obtain the eigenstate with zero energy

$$
\begin{aligned}
\eta_{0}^{l, b}(x) & =\gamma_{l, b}(x)\left(-\frac{d \zeta_{0}^{l+1, b}(x)}{d x}+v_{0}^{l, b} \zeta_{0}^{l+1, b}(x)\right) \\
& =\sqrt{\frac{b^{l+5 / 2}}{2^{l+3 / 2} \Gamma(l+5 / 2)}} \frac{x^{l+2} e^{-b x^{2} / 4}}{\sqrt{b x^{2}-2(l+1)}},
\end{aligned}
$$

as can be checked by direct calculation. Notice that $b x^{2}-2(l+1)>0$ always since $l<-1$ and $b>0$, and therefore $V_{l, b}^{i m}(x)$ and $\eta_{0}^{l, b}(x)$ are defined in the whole interval $(0, \infty)$. Moreover, $\eta_{0}^{l, b}(x)$ has no zeros, and it tends to zero when $x$ goes to 0 and to $\infty$ fast enough to give a square-integrable eigenfunction. In fact, it can be easily checked that

$$
\left(\eta_{0}^{l, b}, \eta_{0}^{l, b}\right)=\int_{0}^{\infty}\left|\eta_{0}^{l, b}(x)\right|^{2} d x=\frac{e^{-l-1}}{2}(-l-1)^{l+3 / 2} \Gamma\left(-l-\frac{3}{2},-l-1\right)
$$


where $\Gamma(\alpha, x)$ denotes the incomplete Gamma function defined by $\Gamma(\alpha, x)=$ $\int_{x}^{\infty} e^{-t} t^{\alpha-1} d t$. The previous formula can be derived by means of the change of variable $b x^{2}=2 t$ and using [39, Formula 8.353.3]:

$$
\Gamma(\alpha, x)=\frac{e^{-x} x^{\alpha}}{\Gamma(1-\alpha)} \int_{0}^{\infty} \frac{e^{-t} t^{-\alpha}}{t+x} d t, \quad \operatorname{Re} \alpha<1, x>0 .
$$

As we can see, the norm of the final eigenfunction depends on $l$, not on $b$, and it takes real values only if $l<-1$, in agreement with the range of application for $l$ previously derived.

\subsection{Radial Coulomb-like potentials}

Let us consider now the family of potentials

$$
V_{l, q}(x)=\frac{2 q}{x}+\frac{l(l+1)}{x^{2}},
$$

where $x \in(0, \infty)$ and $q \neq 0, l$ are real parameters. This family shares several characteristics with that of (83). For a start, each member can be regarded as being part of a pair of shape invariant partner potentials, respect to the transformation law $l \rightarrow l+1[1,9]$. Similarly as before, one can obtain the normalized eigenfunctions (up to a modulus one factor) [36]

$$
\zeta_{k}^{l, q}(x)=\sqrt{\frac{\Gamma(k+1)}{\Gamma(2 l+2+k)}} \frac{2^{l+1}|q|^{l+3 / 2}}{(k+l+1)^{l+2}} x^{l+1} e^{\frac{q x}{k+l+1}} L_{k}^{2 l+1}\left(\frac{-2 q x}{k+l+1}\right) .
$$

These eigenfunctions are square-integrable only in the following circumstances: for $l \in(-3 / 2,-1)$ and $q>0$, only the eigenfunction with $k=0$. For $l \in(-1, \infty)$ and $q<0$, the functions (90) are normalizable for all $k=0,1,2, \ldots$ The normalizable solution in the range $l \in(-3 / 2,-1)$, $q>0$, goes to infinity as $x$ tends to zero, meanwhile all the others go to zero as $x \rightarrow 0$. Again, the reason is the existence of different self-adjoint extensions on the different ranges, being the Hamiltonians $H_{l, q}=-\frac{d^{2}}{d x^{2}}+V_{l, q}(x)$ essentially self-adjoint if $l>-3 / 2$ and $q /(l+1)<0$.

The corresponding eigenvalues to the eigenfunctions (90) for the family (89) are [36]:

$$
E_{k}^{l, q}=-\frac{q^{2}}{(k+l+1)^{2}}, \quad k=0,1,2, \ldots
$$

If $q=-1$ and thus $l>-1$ we recover the spectrum given, for example, in [19]. Compare also with [15, p. 389]. 
EXAmple 7.2. Let us use now Theorem 5.2 with two potentials of the family (89) with different values of $l$. We ask that

$$
V_{l, q}(x)-V_{l-r, q}(x)=\frac{(2 l+1-r) r}{x^{2}}=\frac{1}{\gamma_{l, r}^{2}(x)},
$$

where $r>0$ is to be determined below, so we can choose $\gamma_{l, r}(x)=$ $x / \sqrt{r(2 l+1-r)}$. We will transform one eigenfunction $\zeta_{k}^{l-r, q}(x)$ which satisfies

$$
-\frac{d^{2} \zeta_{k}^{l-r, q}(x)}{d x^{2}}+\left\{V_{l-r, q}(x)+\frac{q^{2}}{(k+l-r+1)^{2}}\right\} \zeta_{k}^{l-r, q}(x)=0,
$$

for some $k=0,1,2, \ldots$, by using some suitable solution of the equation

$$
-\frac{d^{2} \phi_{v}}{d x^{2}}+\left\{V_{l, q}(x)+\frac{q^{2}}{(k+l-r+1)^{2}}\right\} \phi_{v}=0 .
$$

A natural idea is to choose $\phi_{v}(x)$ as one of the eigenfunctions $\zeta_{m}^{l, q}(x)$ of $V_{l, q}(x)$ for certain integer $m$ defined by the condition

$$
E_{m}^{l, q}=-\frac{q^{2}}{(k+l-r+1)^{2}}
$$

whose simplest solution is $m=k-r$. Since $m$ and $k$ are non-negative integers, we have $k \geq r$ and therefore $r$ must be a non-negative integer as well. As in Example 7.1, in order to avoid singularities in the final potential, we have to take $m=0$ and hence $k=r$. Then, we transform the eigenfunction corresponding to the integer $k>0$ of the potential $V_{l-k, q}(x)$, with eigenvalue $-q^{2} /(l+1)^{2}$, by using the ground state of the potential $V_{l, q}(x)$, with the same energy eigenvalue. The original eigenfunction has to be normalizable, so it must be $l-k>-1$ and hence $q<0$, because in the range $l \in(-3 / 2,-1)$ there are only one normalizable eigenfunction and $k>0$. Consequently, $l>k-1 \geq 0$, and both of the initial and intermediate eigenfunctions are square-integrable and go to zero as $x \rightarrow 0$. If we denote $v_{0}^{l, q}(x)=\left(1 / \zeta_{0}^{l, q}(x)\right) d \zeta_{0}^{l, q}(x) / d x$, the image potential reads

$$
V_{l-k, q}(x)-2\left(\frac{v_{0}^{l, q}}{x}+\frac{d v_{0}^{l, q}}{d x}\right)=V_{l-k, q}(x)-\frac{2 q}{(l+1) x}=V_{l-k, q l /(l+1)}(x) .
$$

Correspondingly we find, after some calculations, the final eigenfunction

$$
\eta_{k}^{l, q}(x)=\gamma_{l, k}(x)\left(-\frac{d \zeta_{k}^{l-k, q}(x)}{d x}+v_{0}^{l, q} \zeta_{k}^{l-k, q}(x)\right)=\sqrt{\frac{l}{l+1}} \zeta_{k-1}^{l-k, q l /(l+1)}(x) .
$$


In this way we recover the original potential $V_{l-k, q}(x)$ but with the coupling constant $q$ scaled by the factor $l /(l+1)>0$. This scaling is also reflected in the final eigenfunction, which moreover has $k-1$ instead of $k$, and norm $\sqrt{l /(l+1)}$.

EXAMPLE 7.3. We will consider now the following modified version of the potentials (89):

$$
V_{l, q}(x)=\frac{2 q}{x}+\frac{l(l+1)}{x^{2}}+\frac{q^{2}}{(l+1)^{2}},
$$

where again $x \in(0, \infty)$ and $l, q$ are real parameters. The normalized eigenfunctions are given also by (90), and as before there exist only the normalizable eigenfunction for $k=0$ if $l \in(-3 / 2,-1), q>0$, and in the range $l \in(-1, \infty), q<0$, for all $k=0,1,2, \ldots$ However, the corresponding eigenvalues for the potentials (92) are now

$$
E_{k}^{l, q}=\frac{q^{2}}{(l+1)^{2}}-\frac{q^{2}}{(k+l+1)^{2}}, \quad k=0,1,2, \ldots
$$

As in previous examples, we use two members of the family (92) with different values of $l$. Following Example 7.1, we think of using $V_{l+1, q}(x)$ as the initial potential and $V_{l, q}(x)$ as the intermediate one with $-3 / 2<l<$ -1 . The eigenfunction of the initial potential has to be square-integrable so we must set $q<0$. This means that the intermediate potential $V_{l, q}(x)$ will have no square-integrable eigenfunctions.

One simple way to overcome this difficulty is just to change the sign of $q$ in the intermediate potential, which is what we will do in this Example. The interesting point, however, is that it is even possible to use a non normalizable eigenfunction of $V_{l, q}(x)$ as the intermediate one, leading to physically interesting results. We will see this in the next Example. From the analysis of these two examples it can be shown that the range $l \in$ $(-3 / 2,-1)$ is indeed the only possibility if we restrict $q$ to take the same absolute value in the initial and intermediate potentials.

Now, assuming that $q<0$, we calculate the difference

$$
V_{l,-q}(x)-V_{l+1, q}(x)=\frac{q^{2}}{(l+1)^{2}}-\frac{q^{2}}{(l+2)^{2}}-\frac{2(l+1)}{x^{2}}-\frac{4 q}{x} .
$$

The first two terms coincide with $E_{1}^{l, q}>0$. The third and fourth are always positive for $x \in(0, \infty)$ if $l<-1$ and $q<0$. An appropriate $\gamma(x)$ is 
therefore

$$
\gamma_{l, q}(x)=\frac{x}{\sqrt{\frac{(2 l+3) q^{2} x^{2}}{(l+1)^{2}(l+2)^{2}}-4 q x-2(l+1)}} .
$$

The spectra (93) of two members of the family (92) with values of $l$ differing by one coincide only for the ground state energy. As $E_{0}^{l, q}=0$ for all $l, q$, we will transform the ground state of $V_{l+1, q}(x)$ by using the ground state of $V_{l,-q}(x)$, both of them with zero energy. The final potential is, after some calculations,

$$
\begin{aligned}
V_{l, q}^{i m}(x) & =V_{l+1, q}(x)-2\left(\frac{\gamma_{l, q}^{\prime}}{\gamma_{l, q}} v_{0}^{l,-q}+\frac{d v_{0}^{l,-q}}{d x}\right)+\frac{\gamma_{l, q}^{\prime \prime}}{\gamma_{l, q}} \\
= & \frac{2 q}{x}+\frac{(l+1)(l+2)}{x^{2}}+\frac{q^{2}}{(l+2)^{2}} \\
& +\frac{2(l+1) q\left\{2(l+1)(l+2)^{3}+\left(2 l^{2}+6 l+5\right) q x\right\}}{2(l+1)^{2}(l+2)^{2}(l+1+2 q x) x-(2 l+3) q^{2} x^{3}} \\
& +\frac{4(l+1)^{2}(l+2)^{2}(2 l+3) q^{3} x^{2}}{x\left\{2(l+1)^{2}(l+2)^{2}(l+1+2 q x)-(2 l+3) q^{2} x^{2}\right\}^{2}} \\
& -\frac{2(l+1)^{3}(l+2)^{2} q\left\{\left(2 l^{3}+10 l^{2}+10 l-1\right) q x+4(l+1)^{2}(l+2)^{2}\right\}}{x\left\{2(l+1)^{2}(l+2)^{2}(l+1+2 q x)-(2 l+3) q^{2} x^{2}\right\}^{2}},
\end{aligned}
$$

where $v_{0}^{l,-q}(x)=\left(1 / \zeta_{0}^{l,-q}(x)\right) d \zeta_{0}^{l,-q}(x) / d x$, as usual. The known eigenfunction, with zero energy, of the previous potential is

$$
\begin{aligned}
\eta_{0}^{l, q}(x) & =\gamma_{l, q}(x)\left(-\frac{d \zeta_{0}^{l+1, q}(x)}{d x}+v_{0}^{l,-q} \zeta_{0}^{l+1, q}(x)\right) \\
& =-\frac{2^{l+1}|q|^{l+5 / 2} e^{\frac{q x}{l+2}} x^{l+2}\{(l+1)(l+2)+(2 l+3) q x\}}{(l+1)(l+2)^{l+4} \sqrt{\Gamma(2 l+4)} \sqrt{\frac{(2 l+3) q^{2} x^{2}}{(l+1)^{2}(l+2)^{2}}-4 q x-2(l+1)}} .
\end{aligned}
$$

Since $l \in(-3 / 2,-1)$ and $q<0$, this function has neither zeros nor singularities in $(0, \infty)$. Moreover it is square-integrable, for the integral

$$
\left(\eta_{0}^{l, q}, \eta_{0}^{l, q}\right)=\int_{0}^{\infty}\left|\eta_{0}^{l, q}(x)\right|^{2} d x
$$

becomes after the change of variable $t=2|q| x /(l+2)$,

$$
\frac{1}{2(l+2) \Gamma(2 l+4)}\left\{4(l+1) I_{1}(l)-4(l+1)(2 l+3) I_{2}(l)+(2 l+3)^{2} I_{3}(l)\right\},
$$


where

$$
I_{k}(l)=\int_{0}^{\infty} \frac{e^{-t} t^{2 l+3+k}}{d(l, t)} d t, \quad k=1,2,3,
$$

and $d(l, t)=(3+2 l) t^{2}+8(l+2)(l+1)^{2} t-8(l+1)^{3}$. These integrals converge when $l \in(-3 / 2,-1)$. We have computed numerically the complete expression and checked that it takes positive real values in the same interval. The result is a function strictly increasing with $l$, varying from approximately 0.4 to 1 . Taking into account these properties, the eigenfunction $\eta_{0}^{l, q}(x)$ should be the ground-state of the image potential.

EXAMPLE 7.4. As our final example we will consider the previous one but using a non square-integrable eigenfunction, but without zeros, of the intermediate potential. As sometimes happens for the standard intertwining technique, we will arrive to a physically meaningful image potential (see, for example, $[4,5]$ ).

Consider again the family of potentials (92). We choose $V_{l+1, q}(x)$ as the original potential, with $l \in(-3 / 2,-1)$ and $q<0$. The potential $V_{l, q}(x)$ will be the intermediate one. Their associated spectra coincide just at zero energy, although the corresponding eigenfunction for the intermediate potential is not square-integrable. If we consider the difference

$$
V_{l, q}(x)-V_{l+1, q}(x)=\frac{q^{2}}{(l+1)^{2}}-\frac{q^{2}}{(l+2)^{2}}-\frac{2(l+1)}{x^{2}},
$$

we see again that the first two terms coincide with $E_{1}^{l, q}>0$ and that the third one is always positive for $x \in(0, \infty)$ if $l<-1$, so we can define

$$
\gamma_{l, q}(x)=\frac{x}{\sqrt{\frac{(2 l+3) q^{2} x^{2}}{(l+1)^{2}(l+2)^{2}}-2(l+1)}} .
$$

Now, we transform the ground state of $V_{l+1, q}(x)$ by using the formal mathematical eigenfunction of $V_{l, q}(x)$ with zero eigenvalue, which is not normalizable and has no zeros. The final potential becomes now

$$
\begin{aligned}
& V_{l, q}^{i m}(x)=V_{l+1, q}(x)-2\left(\frac{\gamma_{l, q}^{\prime}}{\gamma_{l, q}} v_{0}^{l, q}+\frac{d v_{0}^{l, q}}{d x}\right)+\frac{\gamma_{l, q}^{\prime \prime}}{\gamma_{l, q}}=\frac{q^{2}}{(l+2)^{2}} \\
& +\frac{2 q}{x}+\frac{(l+1)(l+2)}{x^{2}}-\frac{2(l+1) q\left\{2(l+1)(l+2)^{2}+(2 l+3) q x\right\}}{2(l+1)^{3}(l+2)^{2} x-(2 l+3) q^{2} x^{3}} \\
& +\frac{6(l+1)^{3}(l+2)^{2}(2 l+3) q^{2}}{\left\{2(l+1)^{3}(l+2)^{2}-(2 l+3) q^{2} x^{2}\right\}^{2}},
\end{aligned}
$$


where $v_{0}^{l, q}(x)=\left(1 / \zeta_{0}^{l, q}(x)\right) d \zeta_{0}^{l, q}(x) / d x$. The known eigenfunction with zero energy for the image potential is of the form

$$
\begin{aligned}
\eta_{0}^{l, q}(x) & =\gamma_{l, q}(x)\left(-\frac{d \zeta_{0}^{l+1, q}(x)}{d x}+v_{0}^{l, q} \zeta_{0}^{l+1, q}(x)\right) \\
& =-\frac{2^{l+1}|q|^{l+5 / 2} e^{\frac{q x}{l+2}} x^{l+2}\{(l+1)(l+2)-q x\}}{(l+1)(l+2)^{l+4} \sqrt{\Gamma(2 l+4)} \sqrt{\frac{(2 l+3) q^{2} x^{2}}{(l+1)^{2}(l+2)^{2}}-2(l+1)}} .
\end{aligned}
$$

As $l \in(-3 / 2,-1)$ and $q<0, \eta_{0}^{l, q}(x)$ has no singularities for $x \in(0, \infty)$ but has a zero at the value $x_{0}=(l+1)(l+2) / q>0$. This function is square-integrable, since the integral

$$
\left(\eta_{0}^{l, q}, \eta_{0}^{l, q}\right)=\int_{0}^{\infty}\left|\eta_{0}^{l, q}(x)\right|^{2} d x
$$

becomes after the change of variable $t=2|q| x /(l+2)$

$$
\frac{1}{2(l+2) \Gamma(2 l+4)}\left\{4(l+1)^{2} I_{1}(l)+4(l+1) I_{2}(l)+I_{3}(l)\right\},
$$

where

$$
I_{k}(l)=\int_{0}^{\infty} \frac{e^{-t} t^{2 l+3+k}}{d(l, t)} d t, \quad k=1,2,3,
$$

and now $d(l, t)=(3+2 l) t^{2}-8(l+1)^{3}$. These integrals can be computed explicitly with the aid of [39, Formula 8.389.6]:

$$
\begin{gathered}
\int_{0}^{\infty} \frac{t^{\nu} e^{-\mu t}}{\beta^{2}+t^{2}} d t=\frac{\Gamma(\nu)}{2} \beta^{\nu-1}\left\{e^{i(\mu \beta+(\nu-1) \pi / 2)} \Gamma(1-\nu, i \beta \mu)\right. \\
\left.+e^{-i(\mu \beta+(\nu-1) \pi / 2)} \Gamma(1-\nu,-i \beta \mu)\right\} \\
\operatorname{Re} \beta>0, \operatorname{Re} \mu>0, \operatorname{Re} \nu>-1 .
\end{gathered}
$$

In our case, $\mu=1>0, \beta=\sqrt{-8(l+1)^{3} /(2 l+3)}$ is real and positive for $l \in(-3 / 2,-1)$ and $\nu$ is alternatively $2 l+4,2 l+5$ and $2 l+6$, all of them greater than -1 . The final expression for $\int_{0}^{\infty}\left|\eta_{0}^{l, q}(x)\right|^{2} d x$ is

$$
\frac{4(l+1)^{2} i_{1}(l)+8(l+1)(l+2) i_{2}(l)+2(2 l+5)(l+2) i_{3}(l)}{4(l+2)(2 l+3)},
$$

where

$$
\begin{aligned}
& i_{k}(l)=\beta(l)^{2 l+2+k}\left\{e^{i g(l, k)} \Gamma(-2 l-2-k, i \beta(l))\right. \\
& \left.\quad+e^{-i g(l, k)} \Gamma(-2 l-2-k,-i \beta(l))\right\}, \quad k=1,2,3,
\end{aligned}
$$


being $g(l, k)=\beta(l)+(2 l+2+k) \pi / 2$ and $\beta(l)=\sqrt{-8(l+1)^{3} /(2 l+3)}$. This function is real, positive and strictly decreasing from approximately 3 to 1 with $l \in(-3 / 2,-1)$. Then, the calculated eigenstate should correspond to the first excited state of the final potential. This implies that there should exist a ground state eigenfunction with negative energy eigenvalue.

\section{CONCLUSIONS AND OUTLOOK}

Along this article we have studied several important facts concerning the problem of $A$-related or intertwined Hamiltonians. To this end, we have used simple but powerful group theoretical ideas.

The most important results are the the following. Firstly, we have established the relationship between the finite difference algorithm used in [4] and the transformation group on the set of Riccati equations considered in [27], and we have shown that the first is a particular instance of the second.

Secondly, we have identified the group elements preserving a subset of Riccati equations obtained from the set of Schrödinger-like equations by means of the reduction procedure explained in Section 3. In this way we have generalized the results of the finite difference algorithm to a situation which seems to be new.

Thirdly, we have approached the problem of $A$-related or intertwined Hamiltonians in terms of the transformation group on the set of Riccati equations and the reduction method of Section 3, giving new insight into the nature of the problem.

Finally, we have illustrated by means of some examples the use of the new general Theorems found at Section 5, thus generating potentials for which one eigenfunction and its corresponding eigenvalue are exactly known. As far as we know, some of these potentials have not been considered in the literature until now.

These results induce new interesting questions. For instance, in the case of not constant $\gamma(x)$, we do not know whether it is possible to factorize somehow the initial, intermediate and final Hamiltonians.

Another point concerns new applications of Theorem 5.2. On the one hand, it is an obvious idea to try to use potentials different from the oscillator-like and Coulomb-like potentials treated here. On the other hand, inspired by the Example 7.4, it is natural to consider explicitly other non square-integrable eigenfunctions, without zeros, of the intermediate potential, and see whether they lead to physically meaningful final potentials. This will involve to find the general solution of the intermediate Schrödinger-like equation and to restrict then the discussion to the particular solutions without zeros in the interval of interest, even if they have 
no physical interpretation. This means, in some sense, to adapt to our current method the idea introduced by Mielnik in [40], and developed later in $[41,42,43,44]$, among other articles.

Finally, a finite difference formula has been used by Adler in order to discuss the Bäcklund transformations of the Painlevé equations [34, 35], also related with what are called dressing chains and the well-known Kortewegde Vries equation, see [45, 46] and references therein. Moreover, the Darboux transformation can be generalized by using more than one intermediate eigenfunction of the original problem [47]. Likewise, there exist generalizations of the usual intertwining technique to spaces with dimension greater than one $[48,49,50]$. The natural question is whether there is some relationship between these topics and the transformation group on the set of Riccati equations discussed in this paper.

We hope to provide some answers to these questions in future articles.

\section{ACKNOWLEDGMENTS}

We thank the referee for valuable suggestions and for calling several additional references to our attention. We are also grateful to M. Asorey and B. Mielnik for useful comments and discussions.

A.R. thanks the Spanish Ministerio de Educación y Cultura for a FPI grant, research project PB96-0717. Support of the Spanish DGES (PB96-0717) and CONACYT project 32086-E (México), is also acknowledged. 


\section{REFERENCES}

1. L. Infeld and T.E. Hull, Rev. Mod. Phys. 23 (1951), 21.

2. L.É. Gendenshteïn and I.V. Krive, Soviet Phys. Usp. 28 (1985), 645.

3. J.F. Cariñena, G. Marmo, A.M. Perelomov and M.F. Rañada, Int. J. Mod. Phys. A 13 (1998), 4913.

4. D.J. Fernández, V. Hussin and B. Mielnik, Phys. Lett. A 244 (1998), 309.

5. D.J. Fernández and V. Hussin, J. Phys. A: Math. Gen. 32 (1999), 3603.

6. F. Cooper, A. Khare and U.P. Sukhatme, Phys. Rep. 251 (1995), 267.

7. V.B. Matveev and M.A. Salle, "Darboux Transformations and Solitons," SpringerVerlag, Berlin and Heidelberg, 1991.

8. G. Junker and P. Roy, Ann. Phys. (N.Y.) 270 (1998), 155.

9. J.F. Cariñena and A. Ramos, Rev. Math. Phys. 12 (2000), 1279.

10. J.F. Cariñena and A. Ramos, J. Phys. A: Math. Gen. 33 (2000), 3467.

11. A.A. Andrianov, M.V. Ioffe and V.P. Spiridonov, Phys. Lett. A 174 (1993), 273.

12. A.A. Andrianov, M.V. Ioffe and D.N. Nishnianidze, Phys. Lett. A 201 (1995), 103.

13. A.A. Andrianov, M.V. Ioffe, F. Cannata and J.P. Dedonder, Int. J. Mod. Phys. A 10 (1995), 2683.

14. V.G. Bagrov and B.F. Samsonov, Theor. Math. Phys. 104 (1995), 1051.

15. V.G. Bagrov and B.F. Samsonov, Phys. Part. Nucl. 28 (1997), 374.

16. D.J. Fernández, Int. J. Mod. Phys. A 12 (1997), 171.

17. D.J. Fernández, M.L. Glasser and L.M. Nieto, Phys. Lett. A 240 (1998), 15.

18. A.A. Andrianov, F. Cannata, M.V. Ioffe and D. Nishnianidze, Phys. Lett. A 266 (2000), 341.

19. J.O. Rosas-Ortiz, J. Phys. A: Math. Gen. 31 (1998), 10163.

20. R.L. Anderson, Lett. Math. Phys. 4 (1980), 1.

21. J. Harnad, P. Winternitz and R.L. Anderson, J. Math. Phys. 24 (1983), 1062.

22. M.A. del Olmo, M.A. Rodríguez and P. Winternitz, J. Math. Phys. 27 (1986), 14; 28 (1987), 530.

23. J. Beckers, V. Hussin and P. Winternitz, Lett. Math. Phys. 11 (1986), 81.

24. J. Beckers, V. Hussin and P. Winternitz, J. Math. Phys. 27 (1986), 2217.

25. J. Beckers, L. Gagnon, V. Hussin and P. Winternitz, J. Math. Phys. 31 (1990), 2528.

26. M. Havlíčec, S. Pošta and P. Winternitz, J. Math. Phys. 40 (1999), 3104.

27. J.F. Cariñena and A. Ramos, Int. J. Mod. Phys. A 14 (1999), 1935.

28. B. Mielnik, L.M. Nieto and O. Rosas-Ortiz, Phys. Lett. A 269 (2000), 70.

29. J.F. Cariñena, G. Marmo and J. Nasarre, Int. J. Mod. Phys. A 13 (1998), 3601.

30. F. Calogero, J. Math. Phys. 4 (1963), 427.

31. V.M. Strelchenya, J. Phys. A: Math. Gen. 24 (1991), 4965.

32. P. Libermann and Ch.-M. Marle, "Symplectic Geometry and Analytical Mechanics," Reidel, Dordrecht, 1987.

33. J.F. Cariñena, J. Grabowski and A. Ramos, Act. Appl. Math. 64 (2000), to appear.

34. V.E. Adler, Funct. Anal. and Appl. 27 (1993), 141. 
35. V.E. Adler, Physica D $\mathbf{7 3}$ (1994), 335.

36. J.F. Cariñena and A. Ramos, in preparation.

37. M. Reed and B. Simon, "Methods of Modern Mathematical Physics II: Fourier Analysis, Self-Adjointness," Academic Press, New York, 1975.

38. D.J. Fernández, J. Negro and M.A. del Olmo, Ann. Phys. (N.Y.) 252 (1996), 386.

39. I.S. Gradshteyn and I.M. Ryzhik, "Table of Integrals, Series, and Products," 4th ed., Academic Press, New York, 1965.

40. B. Mielnik, J. Math. Phys. 25 (1984), 3387.

41. D.J. Fernández, Lett. Math. Phys. 8 (1984), 337.

42. M.M. Nieto, Phys. Lett. 145B (1984), 208.

43. Z. Dongpei, J. Phys. A: Math. Gen. 20 (1987), 4331.

44. J.I. Díaz, J. Negro, L.M. Nieto and O. Rosas-Ortiz, J. Phys. A: Math. Gen. 32 (1999), 8447.

45. A.B. Shabat, Inverse Problems 8 (1992), 303.

46. A.P. Veselov and A.B. Shabat, Funct. Anal. Appl. 27 (1993), 81.

47. M.M. Crum, Quart. J. Math. Oxford 6 (1955), 121.

48. A.A. Andrianov, N.V. Borisov and M.V. Ioffe, Theor. Math. Phys. 61 (1984), 1078.

49. A.A. Andrianov, N.V. Borisov and M.V. Ioffe, Phys. Lett. A 105 (1984), 19.

50. A.A. Andrianov, N.V. Borisov, M.V. Ioffe and M.I. Éides, Theor. Math. Phys. 61 (1984), 965. 